\title{
Energy consumption study of channel access modes and modulation schemes of the 2.4 GHz narrowband IEEE 802.15.6
}

\author{
Marwa Boumaiz ${ }^{1}$, Mohammed El Ghazi ${ }^{2}$, Mohammed Fattah $^{3}$, Anas Bouayad ${ }^{4}$, Moulhime El Bekkali $^{5}$ \\ 1,2,4,5 Laboratory of Artificial Intelligence, Data Sciences and Emerging Systems, Sidi Mohamed Ben Abdellah University, \\ Fez, Morocco \\ ${ }^{3}$ IMAGE Laboratory, Moulay Ismail University, Meknes, Morocco
}

\begin{tabular}{l} 
Article Info \\
\hline Article history: \\
Received Feb 18, 2020 \\
Revised Jun 2, 2021 \\
Accepted Jun 13, 2021 \\
\hline
\end{tabular}

\section{Keywords:}

$2.4 \mathrm{GHz}$ modulations Energy efficiency IEEE 802.15.6

Random access Scheduled access WBAN

\begin{abstract}
Energy efficiency is an important challenge for wireless body area networks. Therefore, choosing the channel access modes and modulation schemes that guarantee lower energy consumption is necessary to increase the network lifetime, especially in wireless body area network (WBAN) medical applications. The purpose of this paper is to analyze the network energy consumption in on-body medical applications (which are classified as low data rate, medium data rate, and high data rate applications) for two channel access mechanisms: random and scheduled access modes, and two modulation schemes: differential quadrature phase shift keying (DQPSK) and differential binary phase shift keying (DBPSK), which are supported by the $2.4 \mathrm{GHz}$ band of the Institute of Electrical and Electronics Engineers (IEEE) 802.15.6 standard. The considered on-body area network (BAN) of the study supports two communication scenarios: the line-of-sight transmission and the non-line-of-sight communications, referenced as CM3A and CM3B path loss models respectively. Simulation results have demonstrated that the scheduled access mode based on time-division multiple access (TDMA), and DQPSK are the optimal choices to be made at the media access control (MAC) and physical layer levels respectively, in terms of energy efficiency, in low, medium, and high data rate on-body WBAN applications.
\end{abstract}

This is an open access article under the CC BY-SA license.

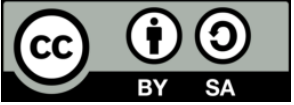

\section{Corresponding Author:}

Marwa Boumaiz

Laboratory of Artificial Intelligence

Data Sciences and Emerging Systems (Ecole Nationale des Sciences Appliquées)

Sidi Mohamed Ben Abdellah University

Avenue My Abdallah Km 5, Route d'Imouzzer, Fès BP 72, Morocco

Email: marwaboumaiz@gmail.com

\section{INTRODUCTION}

Wireless body area networks widely known as WBANs, are composed of tiny body sensors, and wirelessly linked to each other to form a network. This emerging technology is nowadays included in several domains of applications [1]-[3], mainly the medical ones. Non-medical applications are numerous including military, entertainment, assisted living, sports and serious gaming [4], etc. However, when applied in medical services, these systems can facilitate the supervision of simple physiological signs such as blood pressure, electrocardiogram (ECG) [5], and body temperature or be used in more complex operations, such as program changes for pacemakers and implantable defibrillators [6], or even retrieving biokinetic information, in order to collect body movement data, such as acceleration or rotational angular speed [7], [8] and also to adjust the movements of body limbs damaged by car accidents, for example. The gathered data can then be transmitted 
to a gateway, such as a mobile phone, to be sent via Internet or a cellular network to a distant medical database, accessible by authorized healthcare staff for analysis and diagnosis.

One of the major problems faced in WBANs is energy efficiency. Sensor and actuator nodes are usually powered by batteries and it is not always easy to change them, especially when they are implanted inside the human body, which requires surgical operations. Therefore, the design of any WBAN solution must consider this energy aspect at different communication levels. The sources of energy waste are discussed in literature [9] and include collisions, sleep listening, traffic fluctuations, and resource allocation techniques, etc. For this reason, several mechanisms are proposed in literature suggesting methods to improve energy efficiency in WBANs at the mac layer level [10], [11], or proposing routing protocols designed to face the challenge of energy resources scarcity [12], [13]. Furthermore, clean energies are also proposed [14], [15] to compensate for consumed energy in WBANs.

The aim of this paper is to evaluate network energy consumption of aWBAN which is based on the IEEE 802.15.6 at both of media access control (MAC) and physical layer levels, by considering the following MAC configurations: scheduled access mode supported by time-division multiple access (TDMA) and random-access mode supported by carrier sense multiple access with collision avoidance (CSMA/CA). At the physical layer level, the energy consumption is analyzed for two modulation schemes: differential binary phase shift keying (DBPSK) and differential quadrature phase shift keying (DQPSK), these are specific to the 2.4 GHz narrowband of IEEE 802.15.6 standard. Furthermore, two path loss models are also considered for this study, describing the line of sight and non-line of sight transmissions. The following is the structure of the rest of this paper: section 2 presents the WBAN model of study, including the MAC and physical configurations, section 3 highlights the numerical study of network energy consumption for the mentioned access modes and modulation schemes, and section 4 concludes the paper.

\section{PRESENTATION OF THE STUDIED MODEL}

The WBAN model of this study contains ten sensor nodes, one of which is the hub, located towards the patient's belly button [16], [17] as described in Figure 1. The nodes are arranged in a one hop star topology, and intended to supervise most of the vital parameters of body-surface medical applications [18], [19]. The considered energy consumption model assumes: i) the energy consumed by nodes to wake up, ii) the energy required for data transmission, iii) the energy required for data reception, and iv) the energy required for data decoding.

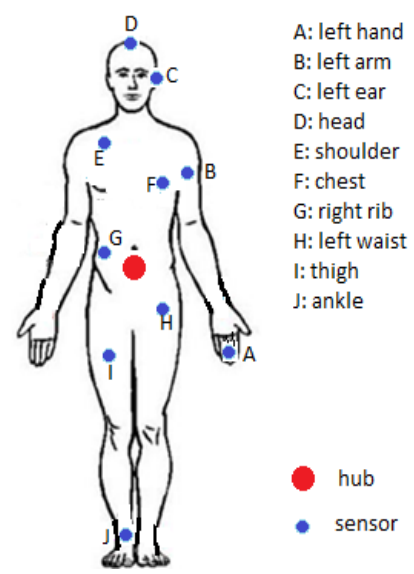

Figure 1. The proposed WBAN model

\subsection{MAC layer}

For time-based resource allocations in a WBAN, the hub is required to time-divide its transmission channel into a set of beacon-bounded and non-beacon-bounded superframes. Nevertheless, under certain circumstances, the hub does not require time referencing of the resource allocations to other sensor nodes in the network and thus is able to function in a non time-based manner, allowing the superframe to be used without beacon boundaries. As a result, the IEEE 802.15.6 standard provides and manages three channel access modes at the MAC layer of a WBAN [20]: i) beacon mode with superframe boundaries, ii) nonbeacon mode with superframe boundaries, and iii) non-beacon mode without superframe boundaries.

This paper addresses the first access mode only. In this mode a beacon is sent by the coordinator during the transmission of an active superframe. The superframe is in turn partitioned into several access 
phases: exclusive access phases (EAP1 and EAP2), random access phases (RAP1 and RAP2), type I/II access phases, and a contention access phase. The exclusive access phases are adopted in the case of urgent traffic; these are the access phases that are used when the data to be transmitted is of high priority. However, the random and contention access phases (RAP1, RAP2 and CAP) are used to send normal traffic that does not have critical emergency conditions. The Type-I/II access phase is used for scheduled and unscheduled bi-link allocations, scheduled uplink and downlink allocations, and type I (not type II) polled and posted allocations.

This paper aims to evaluate WBAN energy consumption for two main access modes: Random access which uses CSMA/CA and scheduled access which supports TDMA protocol. In CSMA/CA, nodes cannot begin transmission until the channel is sensed to be idle, for this reason a backoff counter and a contention window $(\mathrm{CW})$ are used. The backoff counter is a random value initialized by a node and should be between one and the $\mathrm{CW}$, according to the user priority level [21]. The Backoff counter is decreased by one once the cscma/ca slot is idle [22], and once it has reached zero the node can start transmission. However, in TDMA, the bandwidth is divided temporally to distribute the available time over all the nodes of the network, so they can transmit their frames in non overlapping time slots.

\subsection{The $2.4 \mathrm{GHz}$ narrowband (NB) modulation schemes}

The IEEE 802.15.6 standard requires using DBPSK and DQPSK modulations in the narrowband physical layer at $2.4 \mathrm{GHz}$ [20]. Furthermore, choosing the adequate modulation scheme may increase the performance of WBAN applications. In terms of throughput, DQPSK allows the transmission of twice the packets which are transmitted by DBPSK. As for energy consumption, the present paper describes the different behaviors of both modulations. a detailed description of these schemes is presented in our previous work [23], [24].

\subsection{On-body channel models}

The energy consumption analysis of DBPSK and DQPSK modulations is done while considering two modes of propagation, these are the line of sight and non-line of sight signal propagations, defined as $\mathrm{CM} 3 \mathrm{~A}$ and CM3B path loss models respectively [25]. According to CM3A, path loss $L_{\text {path }}$ is given by (1) [17], [25]:

$$
L_{\text {path }}=\operatorname{alog}_{10}(d)+b+N \quad[d B]
$$

where, $L_{\text {path }}(\mathrm{d})$ is the value of path loss at distance $\mathrm{d}(\mathrm{mm})$. a and $\mathrm{b}$ are line fitting coefficients and $\mathrm{N}$ is to be considered a normally distributed value with standard deviation $\sigma \mathrm{N}$.

For non-line of sight communications, CM3B models the path loss as given in (2) [17] [25]:

$$
P L(d)[d B]=-10 \log _{10}\left(P_{0} e^{-m_{0} d}+P_{1}\right)+\sigma_{P} n_{P}
$$

where, PL (d) is the path loss in $\mathrm{dB}$ at a distance d. P0 is a parameter depending on the type of antenna, and the mean losses which take place near the transmitter. P1 represents the mean attenuation in an indoor environment of the components emitted by the human body and reflected towards the receiving antenna. m0 denotes the mean exponential decay in $\mathrm{dB} / \mathrm{cm}$ of the creeping wave components. $\mathrm{Np}$ refers to a random Gaussian parameter having a mean and a unit of zero, and $\sigma p$ refers to the logormal variance which describe the signal variations observed at different points of the body and the room.

For both of CM3A and CM3B models, the studied WBAN model is considered to be subject to hospital room conditions. Therefore, Table 1 shows the parameters values of CM3A and CM3B channel models in a hospital room environment. In this work, body limbs mobility is captured by a temporal variation component, which is thoroughly explained in [26].

Table 1. CM3A and CM3B parameters [17], [25]

\begin{tabular}{ll}
\hline \multicolumn{1}{c}{ Parameter } & \multicolumn{1}{c}{ Value } \\
\hline Frequency & $2.45 \mathrm{GHz}$ \\
$\mathrm{A}$ & 6.6 \\
$\mathrm{~B}$ & 36.1 \\
$\sigma_{\mathrm{N}}$ & 3.8 \\
$P_{0}[\mathrm{~dB}]$ & -25.8 \\
$m_{0}[\mathrm{~dB} / \mathrm{cm}]$ & 2.0 \\
$P_{1}[\mathrm{~dB}]$ & -71.3 \\
$\sigma_{P}[\mathrm{~dB}]$ & 3.6 \\
\hline
\end{tabular}




\section{NUMERICAL STUDY}

\subsection{Energy consumption of random and scheduled access modes}

With a simulation time of $101 \mathrm{~s}$, and a beacon period of 32 time slots, network energy consumption is analyzed for different mac configurations at different transmission data rates, those configurations are as follows: i) Random Access is adopted and its allocations time slots vary from 1 to 3; ii) Scheduled access is used and its periods range from 1 to 3 . Table 2 shows the simulation conditions of channel access modes energy consumption, including the path loss model, the modulation schemes adopted for low, medium and high data rates, the clear channel assessment threshold, the receiver sensitivity (sensitivity of the hub) and other parameters.

\begin{tabular}{ll} 
Table 2. Simulation conditions of channel access modes energy consumption \\
\cline { 2 - 3 } \multicolumn{1}{c}{ Parameter } & \multicolumn{1}{c}{ Value } \\
\hline Simulation time & $101 \mathrm{~s}$ \\
Path loss model & CM3A without Temporal variation \\
Modulation scheme & DBPSK in Low and Medium Data Rate \\
& DQPSK in High Data Rate \\
Transmit power & $-10 \mathrm{dBm}$ \\
Traffic payload & $40 \mathrm{Bytes}$ \\
CCA threshold & $-95 \mathrm{dBm}$ \\
Frequency & $2.45 \mathrm{Ghz}$ \\
Beacon Period Length & 32 time slots \\
Allocation slot length & $10 \mathrm{~s}$ \\
Energy consumed in TX mode & $5.9 \mathrm{~mW}$ \\
Receiver sensitivity $(\mathrm{dBm})$ & -96.5 (for DQPSK) \\
& -104 (for DBPSK) \\
Symbol Rate & 600 \\
Code Rate & $51 / 63$
\end{tabular}

Figures 2, 3 and 4 show network energy consumption in Joules for two access modes: random access wich uses CSMA/CA and scheduled access which supports TDMA, at low (3 kbps), medium (44 kbps) and high $(72 \mathrm{kbps})$ data rate respectively. For the three transmission data rates it can be noticed that the performance of Scheduled Access mode is better in terms of energy efficiency than random access mode. For example, in the case where the transmission rate is equal to $3 \mathrm{kbps}$, the energy consumed by all nodes of the network is around 8.98 joules when the random-access period (RAP) is 1, it reaches 9.09 joules when RAP is equal to 2 and 9.23 when RAP is 3 . This energy consumption is much lower when scheduled access based on TDMA is applied, when the scheduled access period (SAP) is equal to 1 the network's energy consumption is 7.21 joules, it reaches 7.26 when SAP is equal to 2 and 7.32 when SAP is equal to 3 . This good performance of scheduled access compared to random access can be justified by the fact that nodes in the scheduled access mode transmit frames in their dedicated time slots, which avoids collisions and therefore prevents frame retransmissions that cause higher energy consumption. While collisions always take place in the randomaccess mode, which requires packet retransmission. As a result, scheduled access mode is more energy saving, which makes it suitable for low, medium and high data rate on-body medical applications.

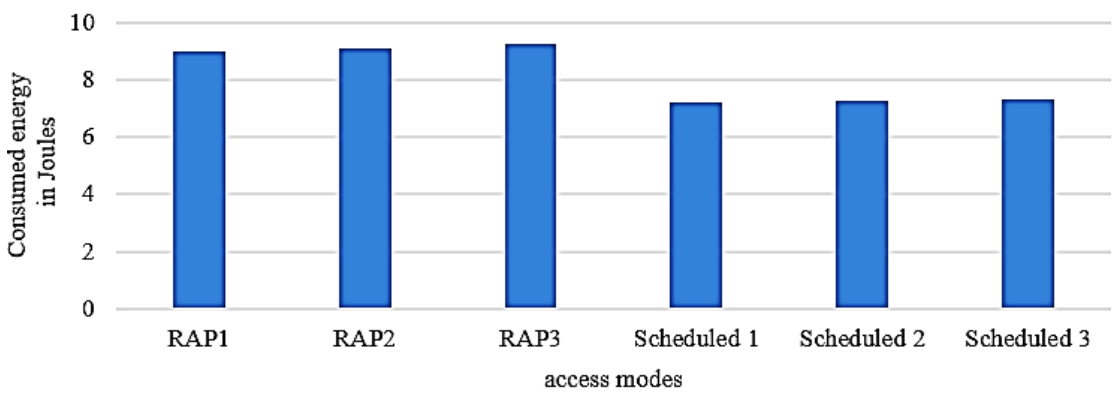

Figure 2. Network energy consumption for different RAP and SAP at low data rate (3kbps) 


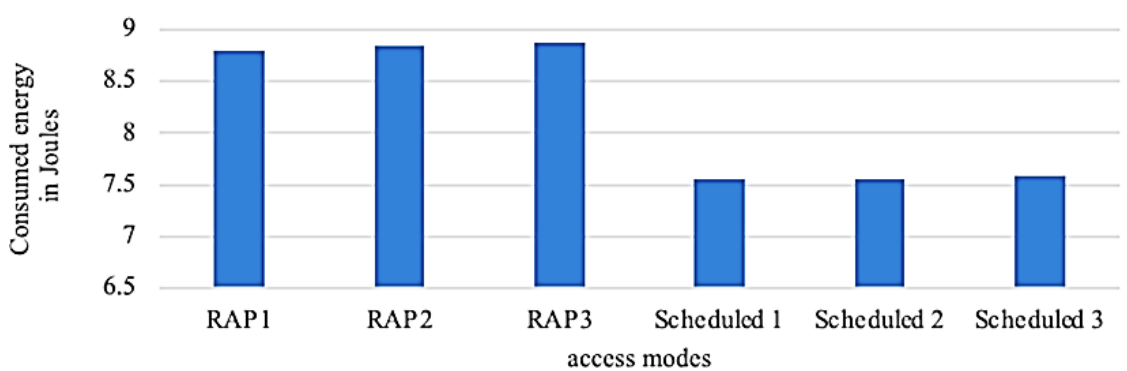

Figure 3. Network energy consumption for different RAP and SAP at Medium data rate (44 kbps)

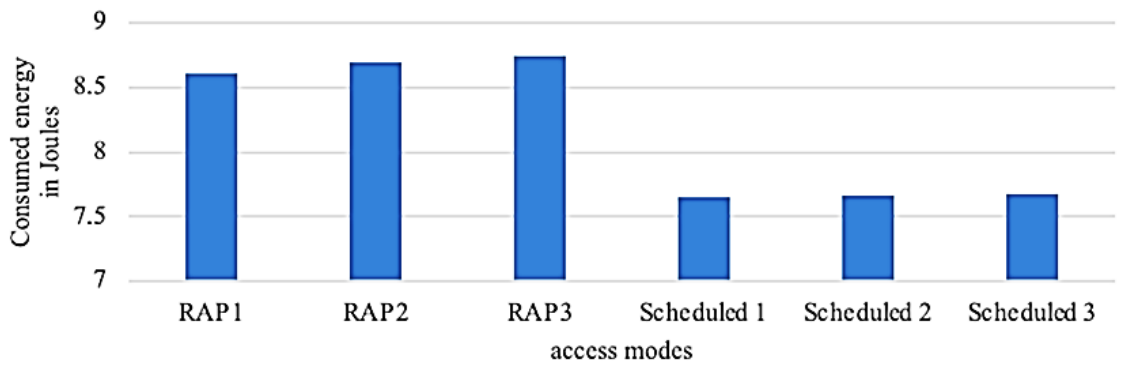

Figure 4. Network energy consumption for different RAP and SAP at High data rate (72 kbps)

\subsection{Energy consumption of DBPSK and DQPSK modulation schemes}

At the physical layer level, the evaluation of network energy consumption is done for the two modulation schemes defined in the $2.4 \mathrm{GHz}$ Narrowband of IEEE 802.15.6 standard. For this purpose, we investigate, through simulations, the network energy consumption, when DBPSK and DQPSK modulations are adopted, considering the two mentioned on-body WBAN channel models: CM3A and CM3B, with and without temporal variation. For this study, a hybrid MAC layer configuration is considered, including two random and 30 scheduled access phases (each node is allocated with three scheduled access intervals) [4], [5]. The other simulation parameters are maintained as those mentioned in Table 2.

Deepak and Babu [27] analyzed the energy consumption for different modulation schemes in coded and uncoded transmissions. In this work, we are interested in coded transmission, which requires the use of a systematic Bose-Chaudhuri-Hocquengham $(\mathrm{BCH})$ code $(\mathrm{n}=63 ; \mathrm{k}=51 ; \mathrm{t}=2)$, to overcome transmission constraints and improve overall reliability [28]. After receiving the physical service data unit (PSDU) from the MAC layer, it is pre-appended with a physical layer convergence protocol (PLCP) header and a PLCP preamble to form the physical layer protocol data unit (PPDU).

If we consider a packet of length $\mathrm{L}$, the required transmission time is defined as follows [27]:

$$
t^{(B C H, M d)}=T_{S}\left[\text { Npreamble }+ \text { NPLCPhdr }+ \text { SPLCPhdr }+\frac{\left[\frac{L}{k}\right] n}{\log _{2} M} \text { SPSDU }\right]
$$

where, Npreamble and N NLCPhdr are the bits in preamble and header of the PPDU. SPLCPhdr and SPSDU are the spreading factors of both of PLCP header and PSDU. The packet of length L is split into k bits blocks and parity check bits of (n-k) are added to create $\left[\frac{L}{k}\right] n$ bits. And $\mathrm{M}$ is the modulation order.

As can be seen in equation 3, the duration of a successful transmission depends essentially on packet length $\mathrm{L}$ and the modulation used. From [27], the energy consumption of bit is highly linked to the transmission time. Hence, to reduce the energy consumption of a WBAN, the transmission time should be reduced, by choosing the optimal modulation scheme and packet length.

Figures 5, 6, 7 and 8 present network energy consumption in joules for DBPSK and DQPSK, when four channel configurations are used (CM3B with/without Temporal variation and CM3A with/without Temporal variations) at low, medium and high data rates corresponding to $3 \mathrm{kbps}, 44 \mathrm{kbps}$ and $72 \mathrm{kbps}$ respectively. In the four figures, it is noticed that network energy consumption is increased when temporal variation phenomenon is considered in channel models (Figures 5 and 7), this is expected as nodes positions may change when body limbs move, which will certainly affect packet reception, resulting in a high packet loss rate, which requires continuous retransmissions. It can also be observed that the consumed energy, for DBPSK and DQPSK when CM3A model is adopted, is reduced in comparison with the case of CM3B. This 
is because CM3B exeriences more path loss than CM3A. i.e the path loss of all the links of the proposed WBAN model is around $71 \mathrm{~dB}$ for CM3B, and is about $55 \mathrm{~dB}$ for CM3A channel model.

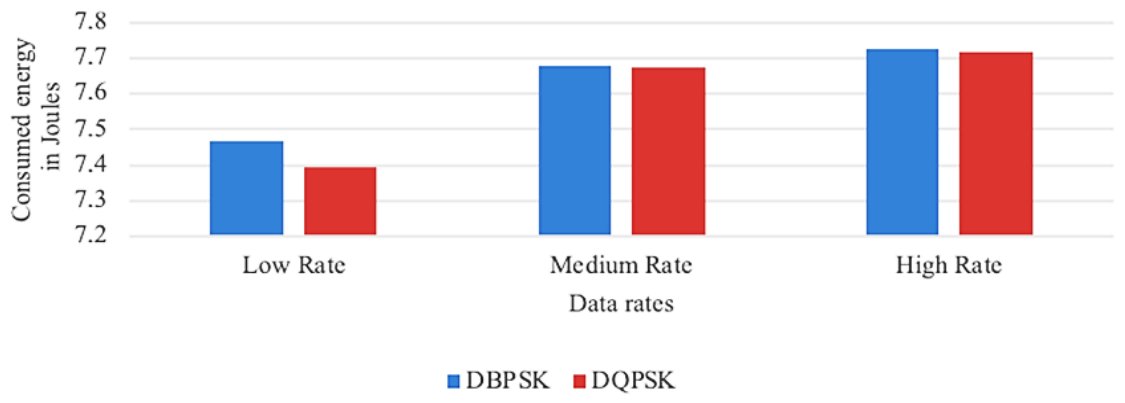

Figure 5. Network consumed energy for DBPSK and DQPSK modulations when CM3B channel model and temporal variation are considered

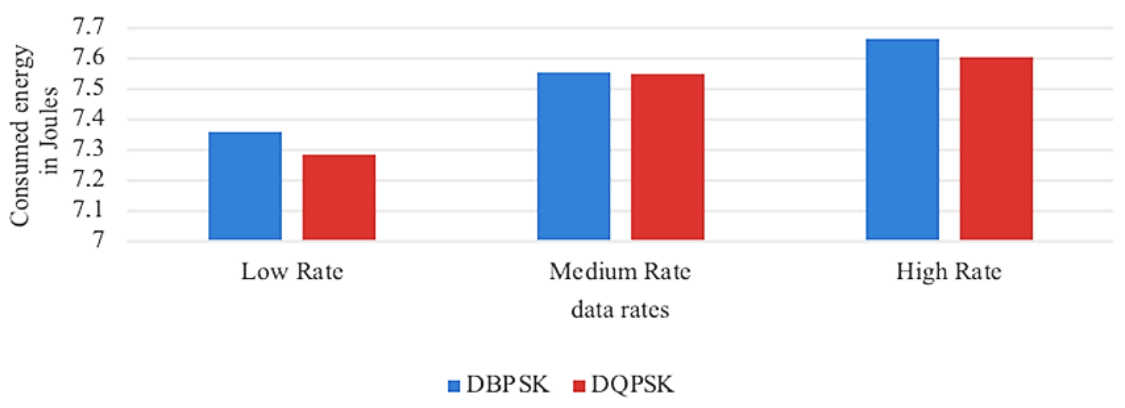

Figure 6. Network consumed energy for DBPSK and DQPSK modulations when CM3B channel model is considered without temporal variation phenomenon

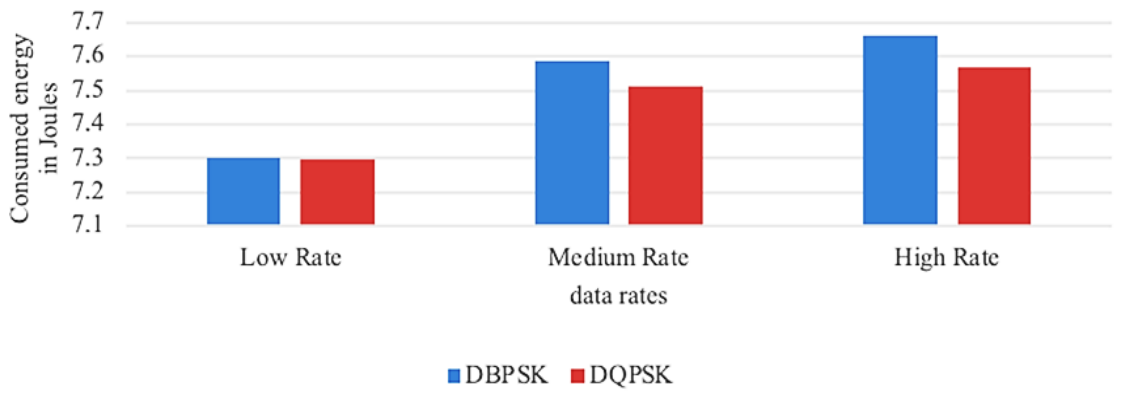

Figure 7. Network consumed energy for DBPSK and DQPSK modulations when CM3A channel model is considered with temporal variation

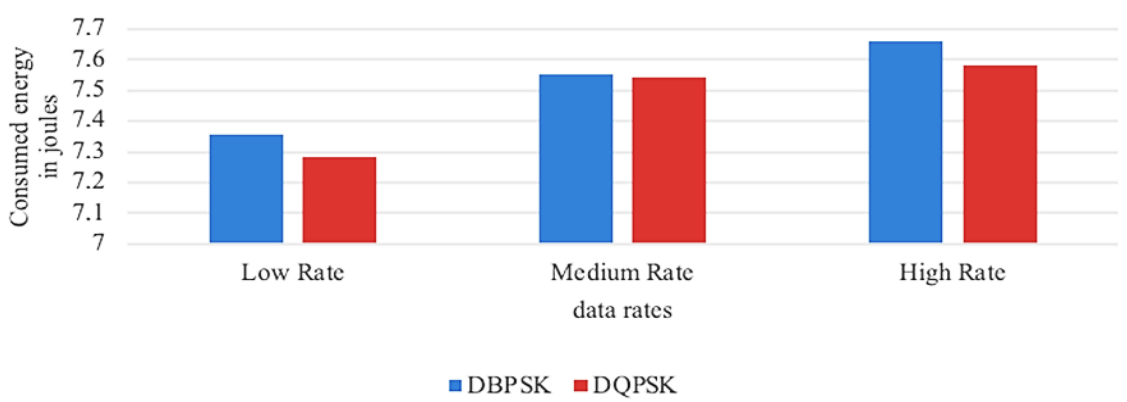

Figure 8. Network consumed energy for DBPSK and DQPSK modulations when CM3A channel model is considered without temporal variation phenomenon 
Regarding modulation shemes, DQPSK is more energy efficient than DBPSK at low, medium and high data rates, because it allows to transmit twice the number of packets that are transmitted with DBPSK. And according to equation 3 the transmission time is related to the modulation order. Hence, as $\mathrm{M}$ increases the transmission time gets lower, which results in a reduced power consumption in higher order modulation schemes. At the physical layer level, to ensure a lower network energy consumtion in the $2.4 \mathrm{GHz} \mathrm{NB}$, DQPSK modulation can be the right choice to be made for low, medium and high data rates on-body medical applications [29]-[31].

\section{CONCLUSION}

In this paper, network energy consumption of an IEEE 802.15.6 WBAN model was studied for two channel access modes, these are the scheduled access mode based on TDMA and the random-access mode based on CSMA/CA, at the mac layer level, and two modulations schemes: DBPSK and DQPSK at the physical layer level. From simulation results, it can be noticed that the scheduled access is more energy efficient than the random one, which makes it a good choice to be made at the mac layer level for low, medium and high data rate on-body WBAN medical applications. As for the physical layer level, DQPSK modulation is more energy saving than DBPSK as it conveys twice of the packets that can be sent with DBPSK, and thus the transmission time is reduced, wich means the consumed energy is also reduced.

The studied WBAN model operates at $2.4 \mathrm{GHz}$. This frequency band is chosen for its worldwild availability and for being the most mature band out of all the bands suggested in the IEEE 802.15.6 standard. Furthermore, the RF technology required for the $2.4 \mathrm{GHz}$ band is already available in the market as it is well used in wifi. However, as we all know, the $2.4 \mathrm{GHz}$ band is already in use by many wireless technologies such as bluetooth, zigbee and Wifi. Hence, the problem of interference between WBANs and these systems (cross interference), or between several adjacent WBANs (mutual interference), may occur. This issue of interference can highly impact the energy efficiency of the network. For this reason, an energy-saving physical and MAC layer configuration must be taken into account in the case of interference (mutual and cross interference), as a perspective to this work.

\section{REFERENCES}

[1] R. Negra, I. Jemili, and A. Belghith, "Wireless body area networks: Applications and technologies," Procedia Computer Science, vol. 83, pp. 1274-1281, 2016, doi: 10.1016/j.procs.2016.04.266.

[2] K. Hasan, K. Biswas, K. Ahmed, N. S. Nafi, and M. Islam, "A comprehensive review of wireless body area network," Journal of Network and Computer Applications, vol. 143, pp. 178-198, 2019, doi: 10.1016/j.jnca.2019.06.016.

[3] D. P. Tobón, T. H. Falk, and M. Maier, "Context awareness in WBANs: a survey on medical and non-medical applications," in IEEE Wireless Communications, vol. 20, no. 4, pp. 30-37, August 2013, doi: 10.1109/MWC.2013.6590048.

[4] T. M. Connolly, E. A. Boyle, E. M. Arthur, T. Hainey, and J. M. Boyle, "Computers \& Education A systematic literature review of empirical evidence on computer games and serious games," Computers \& Education, vol. 59, no. 2, pp. 661-686, 2012, doi: 10.1016/j.compedu.2012.03.004.

[5] J. Jusak and S. S. Mahmoud, "A novel and low processing time ECG security method suitable for sensor node platforms," International Journal of Communication Networks and Information Security, vol. 10, no. 1, pp. 213 222, 2018.

[6] S. Ullah, P. Khan, N. Ullah, S. Saleem, H. Higgins, and K. S. Kwak, "A Review of Wireless Body Area Networks for Medical Applications," International Journal of Communications, Network and System Sciences, vol. 2, no. 8, pp. 797-803, 2009, doi: 10.4236/ijens.2009.28093.

[7] A. Boulemtafes and N. Badache, "Design of wearable health monitoring systems: An overview of techniques and technologies," mHealth Ecosystems and Social Networks in Healthcare, vol 20, pp. 79-94, 2016, doi: 10.1007/9783-319-23341-3_6.

[8] B. Antonescu and S. Basagni, "Wireless body area networks: Challenges, trends and emerging technologies," Proceedings of the 8th International Conference on Body Area Networks, 2013, pp. 1-7, doi: 10.4108/icst.bodynets.2013.253722.

[9] S. Ullah, B. Shen, S. M. R. Islam, P. Khan, S. Saleem, and K. S. Kwak, "A study of MAC protocols for WBANs," Sensors (Basel), vol. 10, no. 1, pp. 128-145, 2010, doi:10.3390/s100100128.

[10] G. Fang and E. Dutkiewicz, "BodyMAC: Energy efficient TDMA-based MAC protocol for wireless body area networks," 2009 9th International Symposium on Communications and Information Technology, 2009, pp. 14551459, doi: 10.1109/ISCIT.2009.5341045.

[11] P. S. Ezhil and K. Selvaradjou, "Performance evaluation of energy efficient MAC protocol for wireless body area network," 2019 IEEE International Conference on System, Computation, Automation and Networking (ICSCAN), Pondicherry, India, 2019, pp. 1-7, doi: 10.1109/ICSCAN.2019.8878807.

[12] K. N. Qureshi, M. U. Bashir, J. Lloret, A. Leon, "Optimized cluster-based dynamic energy-aware routing protocol for wireless sensor networks in agriculture precision,” Journal of Sensors, 2020, doi: 10.1155/2020/9040395. 
[13] J. Anand and D. Sethi, "Comparative analysis of energy efficient routing in WBAN," 2017 3rd International Conference on Computational Intelligence \& Communication Technology (CICT), Ghaziabad, 2017, pp. 1-6, doi: 10.1109/CIACT.2017.7977373.

[14] M. K. M. Rabby, M. S. Alam, and M. S. T. S. A. Shawkat, "A priority based energy harvesting scheme for charging embedded sensor nodes in wireless body area networks," PLoS One, vol. 14, no. 4, 2019, doi: 10.1371/journal.pone.0214716.

[15] M. Boumaiz, et al., "Energy harvesting based WBANs: EH optimization methods," Procedia Computer Science, vol. 151, pp. 1040-1045, 2019, doi: 10.1016/j.procs.2019.04.147.

[16] M. Boumaiz, M. El Ghazi, A. Bouayad, M. Fattah, M. El Bekkali and S. MAzeR, "The impact of transmission power on the performance of a WBAN prone to mutual interference," 2019 International Conference on Systems of Collaboration Big Data, Internet of Things \& Security (SysCoBIoTS), 2019, pp. 1-4, doi: 10.1109/SysCoBIoTS48768.2019.9028035.

[17] M. Boumaiz, M. E. Bekkali, A. Bouayad, and M. Fattah, "The impact of distance between neighboring WBANs on IEEE 802.15.6 performances," 2019 7th Mediterranean Congress of Telecommunications (CMT), 2019, pp. 1-4, doi: 10.1109/CMT.2019.8931341.

[18] F. Di Franco, C. Tachtatzis, R.C. Atkinson, I. Tinnirello, and I. A. Glover , "Channel estimation and transmit power control in wireless body area networks,” IET Wireless Sensor Systems, vol. 5, no. 1, pp. 11-19, 2015, doi: 10.1049/iet-wss.2013.0070.

[19] Kenichi Takizawa et al., "Channel models for wireless body area networks," 2008 30th Annual International Conference of the IEEE Engineering in Medicine and Biology Society, 2008, pp. 1549-1552, doi: 10.1109/IEMBS.2008.4649465.

[20] "IEEE Standard for Local and metropolitan area networks - Part 15.6: Wireless Body Area Networks," in IEEE Std 802.15.6-2012, pp.1-271, 2012, doi: 10.1109/IEEESTD.2012.6161600.

[21] N. Bradai, L. C. Fourati, S. Boudjit, and L. Kamoun, "New priority MAC protocol for wireless body area networks," Proceedings of the 3rd ACM MobiHoc workshop on Pervasive wireless healthcare, 2013, pp. 1-6, doi: $10.1145 / 2491148.2491149$

[22] C. Li, X. Geng, J. Yuan, and T. Sun, "Performance analysis of IEEE 802.15.6 MAC protocol in beacon mode with superframes," KSII Transactions on Internet and Information Systems, vol. 7, no. 5, pp. 1108-1130, 2013, doi: 10.3837/tiis.2013.05.010

[23] E. Shammar, S. Alhomdy, and M. Al-gabri, "Mutual interference mitigation schemes on wireless body area networks ( WBANs ): A survey," International Journal of Computing and Technology, vol. 5, no. 10, pp. 137-147, 2019.

[24] M. Boumaiz, M. El Ghazi, S. Mazer, M. El Bekkali, A. Bouayad, and M. Fattah, "Performance analysis of DQPSK and DBPSK modulation schemes for a scheduled access phase based wireless body area network," 2018 9th International Symposium on Signal, Image, Video and Communications (ISIVC), 2018, pp. 163-167, doi: 10.1109/ISIVC.2018.8709232.

[25] K. Y. Yazdandoost, "Channel Modeling Subcommittee Report,” IEEE P802.15 Networks, vol. 802, 2009.

[26] A. Boulis, "Castalia: A simulator for wireless sensor networks and body area networks," castalia. npc. nicta. com. au, pp. 79, 2010.

[27] K. S. Deepak and A. V. Babu, "Energy consumption analysis of modulation schemes in IEEE 802.15.6-based wireless body area networks," EURASIP Journal on Wireless Communications and Networking, vol. 2016, no. 1, pp.1-14, 2016, doi: 10.1186/s13638-016-0682-5.

[28] P. Mathew, L. Augustine, D. Kushwaha, D. Vivian and D. Selvakumar, "Hardware implementation of NB PHY baseband transceiver for IEEE 802.15.6 WBAN," 2014 International Conference on Medical Imaging, m-Health and Emerging Communication Systems (MedCom), 2014, pp. 64-71, doi: 10.1109/MedCom.2014.7005977.

[29] A. Maroua and F. Mohammed, "Characterization of Ultra Wide Band indoor propagation," 2019 7th Mediterranean Congress of Telecommunications (CMT), 2019, pp. 1-4, doi: 10.1109/CMT.2019.8931367.

[30] A. Es-Saqy, et al., "28 GHz balanced pHEMT VCO with low phase noise and high output power performance for 5G mm-wave systems," International Journal of Electrical and Computer Engineering, vol. 10, no. 5, pp. 46234630, 2020, doi: 10.11591/ijece.v11i2.pp1036-1042.

[31] A. Es-saqy, M. Abata, M. Fattah, S. Mazer, M. Mehdi, and M. El Bekkali, "Study and design of a MMIC voltage controlled oscillator for 5G mm-wave band applications," International Journal of Advanced Trends in Computer Science and Engineering, vol. 9, no. 2, pp. 2124-2129, 2020, doi: 10.30534/ijatcse/2020/186922020. 